\title{
On the spectral investigation of the scattering problem for some version of one-dimensional Schrödinger equation with turning point
}

\author{
Zaki FA El-Raheem ${ }^{1 *}$ and AH Nasser ${ }^{2}$
}

\section{*Correspondence:}

zaki55@Alex-Sci.edu.eg

'Department of Mathematics,

Faculty of Education, Alexandria

University, Alexandria, Egypt

Full list of author information is

available at the end of the article

\section{黛 Springer}

\begin{abstract}
In this paper we introduce and investigate the eigenvalues and the normalizing numbers as well as the scattering function for some version of the one-dimensional Schrödinger equation with turning point on the half line. MSC: 58C 40; 34L25

Keywords: initial value problem; the eigenvalues; normalizing numbers; scattering function; asymptotic formula
\end{abstract}

\section{Introduction}

The solution of many problems of mathematical physics are reduced to the spectral investigation of a differential operator. The differential operator is called regular if its domain is finite and its coefficients are continuous, otherwise it is called a singular differential operator. The Sturm-Liouville theory occupies a central position in the spectral theory of regular operator. During the development of quantum mechanics there was an increase in the interest of spectral theory of singular operators, on which we will restrict our attention. The first basic role in the development of the spectral theory of singular operators dates back to Titchmarsh [1]. He gave a new approach in the spectral theory of singular differential operator of the second order by using contour integration. Also Levitan [2] gave a new method, he obtained the eigenfunction expansion in an infinite interval by taking the limit of a regular case. In the last 35 or so years, due to the needs of mathematical physics, in particular, quantum mechanics, the question of solving various spectral problems with explosive factor has appeared in the study of geophysics and electromagnetic fields; see [3, 4]. The spectral theory of differential operators with explosive factor is studied by Tikhonov [5], Gasymov [6]. For earlier results on various aspects of solvability theory of boundary value problems and spectral theory in the half line case, the situation closely related to the principal topic of this paper, we refer, for instance, to [7-10]. Notice that the paper [11] presented an approximate construction of the Jost function for some Sturm-Liouville boundary value problem in the case $\rho(x)=1$ by means of the collocation method. In the present paper we introduce and investigate the eigenvalues and the normalizing numbers as well as the scattering function for some version of the onedimensional Schrödinger equation with turning point on the half line as in (1.1), (1.2). In $[12,13]$, and [14] the weight functions introduced are considered as applications of the

@2014 El-Raheem and Nasser; licensee Springer. This is an Open Access article distributed under the terms of the Creative Commons Attribution License (http://creativecommons.org/licenses/by/2.0), which permits unrestricted use, distribution, and reproduction in any medium, provided the original work is properly cited. 
discontinuous wave speed problem on a non-homogeneous medium as in our case, while the introduction of the weight function $\rho(x)$ which is given by (1.3) as \pm signs causes an excess of analytical difficulties. In [15] the author studied the spectral property in a finite interval, while in the present work we consider the half line which gives rise both to a continuous and a discrete spectrum; the latter is treated by the scattering function. In [16] the author considered the weight function of the form

$$
\rho(x)= \begin{cases}\alpha^{2} ; & \operatorname{Im} \alpha \neq 0,0 \leq x \leq a<\pi, \\ 1 ; & a<x \leq \pi,\end{cases}
$$

and the spectra were both continuous and discrete as in our problem. We must notice that the result of this paper is a starting point in calculating the regularized trace formula and solving the inverse scattering problem, which will be investigated later on.

Consider the initial value problem

$$
\begin{aligned}
& -y^{\prime \prime}+q(x) y=\mu \rho(x) y, \quad 0 \leq x<\infty, \\
& y^{\prime}(0)-h y(0)=0, \quad h>0,
\end{aligned}
$$

where

$$
\rho(x)= \begin{cases}-1 ; & 0 \leq x \leq 1, \\ 1 ; & 1<x<\infty\end{cases}
$$

$q(x)$ is a finite real valued function which satisfies

$$
\int_{0}^{\infty}(1+x)|q(x)| d x<\infty
$$

and $\mu$ is a complex spectral parameter. To study the eigenvalues of (1.1)-(1.2), we first consider the case when $q(x) \equiv 0$ and $h=0$.

For $q(x) \equiv 0$ and $h=0$ problem (1.1)-(1.2) takes the form

$$
\begin{aligned}
& -y^{\prime \prime}=\mu \rho(x) y, \quad 0 \leq x \leq \infty, \\
& y^{\prime}(0)=0, \\
& \mu=\lambda^{2} .
\end{aligned}
$$

From now on we consider $\operatorname{Im} \lambda \geq 0$ because according to (1.6) $\mu$ covers all the complex plane. Denote by $\varphi_{o}(x, \lambda)$ the solution of $(1.4)$ with the initial conditions $\varphi_{o}(0, \lambda)=1$, $\varphi_{o}^{\prime}(0, \lambda)=0$. According to (1.3), (1.4) is equivalent to the two equations

$$
\begin{aligned}
& -y^{\prime \prime}=-\lambda^{2} y, \quad 0 \leq x<1, \\
& -y^{\prime \prime}=\lambda^{2} y, \quad 1<x \leq \infty .
\end{aligned}
$$

It is easy to see that

$$
\varphi_{o}(x, \lambda)= \begin{cases}\cosh \lambda x ; & 0 \leq x<1, \\ a_{o}(\lambda) e^{i \lambda x}+b_{o}(\lambda) e^{-i \lambda x} ; & 1<x<\infty,\end{cases}
$$


where $a_{o}(\lambda), b_{o}(\lambda)$ are calculated from the requirements $\varphi_{o}(1-0, \lambda)=\varphi_{o}(1+0, \lambda)$ and $\varphi_{o}^{\prime}(1-$ $0, \lambda)=\varphi_{o}^{\prime}(1+0, \lambda)$, so that $(1.8)$ takes the form

$$
\varphi_{o}(x, \lambda)= \begin{cases}\cosh \lambda x ; & 0 \leq x<1, \\ \frac{e^{-i \lambda}}{2}(\cosh \lambda-i \sinh \lambda) e^{i \lambda x}+\frac{e^{i \lambda}}{2}(\cosh \lambda+i \sinh \lambda) e^{-i \lambda x} ; & 1<x<\infty .\end{cases}
$$

For $\operatorname{Im} \lambda=0$, the function $\varphi_{o}(x, \lambda)$ does not belong to $L_{2}(0, \infty)$ also, for $\operatorname{Im} \lambda>0, e^{i \lambda x} \rightarrow 0$ as $x \rightarrow \infty$ whereas $e^{-i \lambda x} \rightarrow \infty$ as $x \rightarrow \infty$, so that it is convenient to consider

$$
e^{i \lambda}(\cosh \lambda+i \sinh \lambda)=0
$$

as the equation of the eigenvalues $\mu_{o}=\lambda_{o}^{2}$.

From this we have $\lambda_{o}=\left(n+\frac{1}{4}\right) \pi i, n=0,1, \pm 1, \pm 2, \ldots$ or

$$
\mu_{n}^{o}=-\left(n+\frac{1}{4}\right)^{2} \pi^{2}, \quad n=1,2, \ldots
$$

Together with the solution $\varphi_{o}(x, \lambda)$ of $(1.4)$ we introduce the second solution $f_{o}(x, \lambda)$, which is known as the Jost solution. This solution is defined by the condition

$$
f_{o}(x, \lambda) \approx e^{i \lambda x}, \quad x \rightarrow \infty
$$

With the aid of (1.7), we have

$$
f_{o}(x, \lambda)= \begin{cases}c_{o}(\lambda) \cosh \lambda x+d_{o}(\lambda) \sinh \lambda x, & 0 \leq x<1 \\ e^{i \lambda x}, & 1<x<\infty\end{cases}
$$

where the coefficients $c_{o}(\lambda), d_{o}(\lambda)$ are calculated from the requirements $f_{o}(1-0, \lambda)=f_{o}(1+$ $0, \lambda)$ and $f_{o}^{\prime}(1-0, \lambda)=f_{o}^{\prime}(1+0, \lambda)$, and the solution becomes

$$
f_{o}(x, \lambda)= \begin{cases}e^{i \lambda}(\cosh \lambda-i \sinh \lambda) \cosh \lambda x & \\ \quad+e^{i \lambda}(i \cosh \lambda-\sinh \lambda) \sinh \lambda x, & 0 \leq x<1, \\ e^{i \lambda x}, & 1<x<\infty .\end{cases}
$$

It should be noted, here, that the equation of the eigenvalues can be obtained, also, from the condition that the solution $f_{o}(x, \lambda) \in L_{2}(0, \infty ; \rho)$; this condition implies that $f_{o}^{\prime}(0, \lambda)=0$, which is the same as (1.10).

Now for $q(x) \neq 0, h \neq 0$ we denote by $f(x, \lambda)$ the solution of (1.1) which satisfies the condition

$$
f(x, \lambda) \approx e^{i \lambda x}, \quad x \rightarrow \infty,-\infty<\lambda<\infty .
$$

For $x>1$, (1.1) takes the form $-y^{\prime \prime}+q(x) y=\lambda^{2} y$, and in the following, we study its solution and the related spectrum. From [4] this solution has the following representation:

$$
f(x, \lambda)=e^{i \lambda x}+\int_{x}^{\infty} K(x, t) e^{i \lambda t} d t, \quad 1<x<\infty
$$

where $\operatorname{Im} \lambda \geq 0, K(x, x)=\frac{1}{2} \int_{0}^{x} q(t) d t, 1<x<\infty$. 
For $0 \leq x \leq 1$, the solution $f(x, \lambda)$ has the form

$$
f(x, \lambda)= \begin{cases}a(\lambda) \varphi(x, \lambda)+b(\lambda) \theta(x, \lambda), & 0 \leq x \leq 1 \\ e^{i \lambda x}+\int_{x}^{\infty} K(x, t) e^{i \lambda t} d t, & 1<x<\infty\end{cases}
$$

where $\varphi(x, \lambda), \theta(x, \lambda)$ is the fundamental system of solutions of (1.1) subject to the initial conditions

$$
\begin{array}{ll}
\varphi(0, \lambda)=1, & \varphi^{\prime}(0, \lambda)=h, \\
\theta(0, \lambda)=0, & \theta^{\prime}(0, \lambda)=1,
\end{array}
$$

where the coefficients $a(\lambda), b(\lambda)$ are calculated from the requirements $f(1-0, \lambda)=f(1+$ $0, \lambda), f^{\prime}(1-0, \lambda)=f^{\prime}(1+0, \lambda)$, from which

$$
\begin{aligned}
& a(\lambda)=f(1, \lambda) \theta^{\prime}(1, \lambda)-f^{\prime}(1, \lambda) \theta(1, \lambda), \\
& b(\lambda)=f^{\prime}(1, \lambda) \varphi(1, \lambda)-f(1, \lambda) \varphi^{\prime}(1, \lambda) .
\end{aligned}
$$

Further, (1.1), for $0 \leq x \leq 1$, takes the form $-y^{\prime \prime}+q(x) y=-\lambda^{2} y$, and the fundamental system of solution of this follows from [4, p.18] by the representation

$$
\begin{aligned}
& \varphi(x, \lambda)=\frac{\sinh \lambda x}{\lambda}+\int_{0}^{x} B(x, t) \frac{\sinh \lambda t}{\lambda} d t, \\
& A(x, x)=\frac{1}{2} \int_{0}^{x} q(t) d t, \quad A(x, 0)=0, \quad A(0,0)=h, \\
& \theta(x, \lambda)=\cosh \lambda x+\int_{0}^{x} A(x, t) \cosh \lambda t d t, \\
& B(x, x)=\frac{1}{2} \int_{0}^{x} q(t) d t,\left.\quad \frac{\partial B}{\partial t}\right|_{t=0}=0, \quad B(0,0)=1 .
\end{aligned}
$$

Now we find the characteristic equation of the eigenvalues of (1.1)-(1.2). Since the solution (1.15) belongs to $L_{2}(0, \infty), \operatorname{Im} \lambda>0$ it follows that, for $\mu=\lambda^{2}$ to be an eigenvalue, it must satisfy the initial condition (1.2), namely

$$
f^{\prime}(0, \lambda)-h f(0, \lambda)=0
$$

From (1.15) and (1.16) we have

$$
f^{\prime}(0, \lambda)-h f(0, \lambda)=b(\lambda)=f^{\prime}(1, \lambda) \varphi(1, \lambda)-f(1, \lambda) \varphi^{\prime}(1, \lambda)
$$

In the following lemmas we study some properties of the eigenvalues of problem (1.1)-(1.2).

Lemma 1.1 Under the conditions $q(x)>0(0<x<\infty)$, the roots of $(1.20)$, for $\operatorname{Im} \lambda>0$, are simple and lie only on the imaginary axis.

Proof Let $\lambda_{o}$, where $\operatorname{Im} \lambda_{o}>0$, be a zero of the function $f^{\prime}(0, \lambda)-h f(0, \lambda)$, so that

$$
f^{\prime}\left(0, \lambda_{o}\right)-h f\left(0, \lambda_{o}\right)=0 \text {. }
$$


We prove that $\lambda_{o}=i \tau_{o}, \tau_{o}>0$. Since $f\left(x, \lambda_{o}\right)$ is a solution of (1.1) we have

$$
-f^{\prime \prime}\left(x, \lambda_{o}\right)+q(x) f\left(x, \lambda_{o}\right)=\lambda_{o}^{2} \rho(x) f\left(x, \lambda_{o}\right)
$$

multiplying both sides of this by $\overline{f\left(x, \lambda_{o}\right)}$ and integrating both sides from 0 to $\infty$, we have

$$
-\int_{0}^{\infty} f^{\prime \prime}\left(x, \lambda_{o}\right) \overline{f\left(x, \lambda_{o}\right)} d x+\int_{0}^{\infty} q(x)\left|f\left(x, \lambda_{o}\right)\right|^{2} d x=\lambda_{o}^{2} \int_{0}^{\infty} \rho(x)\left|f\left(x, \lambda_{o}\right)\right|^{2} d x
$$

Integrating the first integral by parts and using (1.22), (1.15) we obtain

$$
\lambda_{o}^{2}=\frac{\int_{0}^{\infty}\left\{\left|f^{\prime}\left(x, \lambda_{o}\right)\right|^{2}+q(x)\left|f\left(x, \lambda_{o}\right)\right|^{2}\right\} d x+h\left|f\left(0, \lambda_{o}\right)\right|^{2}}{\int_{0}^{\infty} \rho(x)\left|f\left(x, \lambda_{o}\right)\right|^{2} d x}
$$

where $\int_{0}^{\infty} \rho(x)\left|f\left(x, \lambda_{o}\right)\right|^{2} d x \neq 0$, from which we deduce that $\lambda_{o}^{2}$ is real and hence $\lambda_{o}$ is pure imaginary. We turn now to the proof that the roots are simple from (1.22), this is carried out by proving that $f^{\prime}(0, \lambda)-h f(0, \lambda)=0$ implies $\left[\dot{f}^{\prime}(0, \lambda)-h \dot{f}(0, \lambda)\right] \neq 0$, where 'dot' denotes differentiation with respect to $\lambda$.

Integrating the difference $[\dot{f}(x, \lambda) \times(1.23)]-\left[f(x, \lambda) \times \frac{d}{d \lambda}(1.23)\right]$ with respect to $x$ from 0 to $\infty$ and using (1.20) we get after some calculation that

$$
f(0, \lambda)\left[\dot{f}^{\prime}(0, \lambda)-h \dot{f}(0, \lambda)\right]=2 \lambda \int_{0}^{\infty} \rho(x) f^{2}(x, \lambda) d x
$$

We prove the reality of $f(x, \lambda)$.

For $x>1, \lambda=i \tau$ the function $f(x, \lambda)=e^{-\tau x}+\int_{x}^{\infty} K(x, t) e^{-\tau t} d t$ is real because reality of $K(x, t)$ comes from the reality of $q(x)$.

To prove that, for $0 \leq x<1$, we observe that $\varphi$ and $\theta$ are real. Let $\lambda=i \tau$; since $\varphi(x, \lambda)$ is a solution of (1.1)-(1.2), we have

$$
-\varphi^{\prime \prime}(x, \lambda)+q(x) \varphi(x, \lambda)=-\tau^{2} \rho(x) \varphi(x, \lambda), \quad \varphi(0, \lambda)=1, \quad \varphi^{\prime}(0, \lambda)=h .
$$

Taking the conjugate of (1.26) we have

$$
-\overline{\varphi^{\prime \prime}(x, \lambda)}+q(x) \overline{\varphi(x, \lambda)}=-\tau^{2} \rho(x) \overline{\varphi(x, \lambda)}, \quad \overline{\varphi(0, \lambda)}=1, \quad \overline{\varphi^{\prime}(0, \lambda)}=h .
$$

It is clear, from (1.26) and (1.27), that $\varphi(x, \lambda)=\overline{\varphi(x, \lambda)}$. In a similar way we can prove that $\theta(x, \lambda)$ is also real so that the solution $f(x, \lambda)$ for $0 \leq x<1$ is real from which we have $f^{2}(x, \lambda)=|f(x, \lambda)|^{2}$ and (1.25) takes the form

$$
f(0, \lambda)\left[\dot{f}^{\prime}(0, \lambda)-h \dot{f}(0, \lambda)\right]=2 \lambda \int_{0}^{\infty} \rho(x)|f(x, \lambda)|^{2} d x
$$

From $(1.28)$ we see that $\frac{d}{d \lambda}\left[f^{\prime}(0, \lambda)-h f(0, \lambda)\right] \neq 0$, which completes the proof.

Remark 1 For $\operatorname{Im} \lambda_{n}>0$ and $f^{\prime}\left(0, \lambda_{n}\right)-h f\left(0, \lambda_{n}\right)=0$, the function $f\left(0, \lambda_{n}\right)$ is the eigenfunction of problem (1.1)-(1.2) that corresponds to the negative eigenvalues $\mu_{n}=\lambda_{n}^{2}=-\chi_{n}^{2}$. 
Lemma 1.2 For all $\operatorname{Re} \lambda \neq 0$ the function $f^{\prime}(0, \lambda)-h f(0, \lambda)$ does not tend to zero, i.e.

$$
f^{\prime}(0, \lambda)-h f(0, \lambda) \neq 0, \quad \operatorname{Re} \lambda \neq 0, \quad-\infty<\lambda<\infty .
$$

Proof Since the function $f(x, \lambda)$ is the solution of $(1.1), f(x,-\lambda)$ is also a solution, and it can be shown that these two solutions are linearly independent and their Wronskian is

$$
W[f(x, \lambda), f(x,-\lambda)]=-2 i \lambda,
$$

so that $W[f(x, \lambda), f(x,-\lambda)] \neq 0$, for $\operatorname{Re} \lambda \neq 0$, so that $f(x, \lambda)$ and $f(x,-\lambda)$ is a fundamental system of solutions of (1.1). In particular, putting $x=0$ into (1.30) we have

$$
f(0, \lambda) f^{\prime}(0,-\lambda)-f^{\prime}(0, \lambda) f(0,-\lambda)=-2 i \lambda
$$

To prove that $f^{\prime}(0, \lambda)-h f(0, \lambda) \neq 0, \operatorname{Re} \lambda \neq 0,-\infty<\lambda<\infty$, assume to the contrary i.e. $f^{\prime}(0, \lambda)-h f(0, \lambda)=0, \operatorname{Re} \lambda \neq 0,-\infty<\lambda<\infty$. From (1.31) and (1.20) we reach to contradiction to the assumption, and, consequently, we deduce that $f^{\prime}(0, \lambda)-h f(0, \lambda) \neq 0, \operatorname{Re} \lambda \neq 0$, $-\infty<\lambda<\infty$. Notice that $\overline{f(x, \lambda)}=f(x,-\lambda)$.

Lemma 1.3 For all $\operatorname{Re} \lambda \neq 0$ the following equality holds:

$$
\frac{2 i \lambda \varphi(x, \lambda)}{f^{\prime}(0, \lambda)-h f(0, \lambda)}=f(x,-\lambda)-S(\lambda) f(x, \lambda)
$$

where $\varphi(x, \lambda)$ is the solution of problem (1.1)-(1.2) and the function

$$
S(\lambda)=\frac{f^{\prime}(0,-\lambda)-h f(0,-\lambda)}{f^{\prime}(0, \lambda)-h f(0, \lambda)}
$$

satisfies the properties

$$
\overline{S(\lambda)}=S(-\lambda), \quad|S(\lambda)|=1, \quad-\infty<\lambda<\infty .
$$

It should be noted here that the function $S(\lambda)$ defined by $(1.33)$ is called the scattering function of problem (1.1)-(1.2) and the function $f^{\prime}(0, \lambda)-h f(0, \lambda)$ is called the denominator of $S(\lambda)$.

Proof As mentioned before (1.30) for all $\operatorname{Re} \lambda \neq 0, f(x, \lambda)$ and $f(x,-\lambda)$ is a fundamental system of solutions of (1.1)-(1.2), so that any linear combination of them is again a solution of (1.1)-(1.2):

$$
\varphi(x, \lambda)=A(\lambda) f(x, \lambda)+B(\lambda) f(x,-\lambda)
$$

where $A(\lambda), B(\lambda)$ are calculated from the initial conditions $\varphi(0, \lambda)=1, \varphi^{\prime}(0, \lambda)=h$ in the form

$$
A(\lambda)=\frac{f^{\prime}(0,-\lambda)-h f(0,-\lambda)}{-2 i \lambda}, \quad B(\lambda)=\frac{f^{\prime}(0, \lambda)-h f(0, \lambda)}{-2 i \lambda}
$$


Substituting (1.36) into (1.35) we arrive at the required formula (1.32). Further, since $\overline{f(x, \lambda)}=f(x,-\lambda)$, it follows from (1.33) that

$$
S(\lambda)=\frac{f^{\prime}(0,-\lambda)-h f(0,-\lambda)}{f^{\prime}(0, \lambda)-h f(0, \lambda)}=\frac{\overline{f^{\prime}(0, \lambda)-h f(0, \lambda)}}{f^{\prime}(0, \lambda)-h f(0, \lambda)},
$$

from which we have

$$
|S(\lambda)|=\frac{\left|\overline{f^{\prime}(0, \lambda)-h f(0, \lambda)}\right|}{\left|f^{\prime}(0, \lambda)-h f(0, \lambda)\right|}=1,
$$

and

$$
\overline{S(\lambda)}=\overline{\left(\frac{f^{\prime}(0,-\lambda)-h f(0,-\lambda)}{f^{\prime}(0, \lambda)-h f(0, \lambda)}\right)}=\frac{f^{\prime}(0, \lambda)-h f(0, \lambda)}{f^{\prime}(0,-\lambda)-h f(0,-\lambda)}=S(-\lambda) .
$$

\section{The asymptotic formulas of eigenvalues and normalizing numbers}

The eigenvalues $\mu=\lambda^{2}$ of problem (1.1)-(1.2) are the roots of the equation

$$
f^{\prime}(0, \lambda)-h f(0, \lambda)=0, \quad \operatorname{Im} \lambda>0 .
$$

In the following we prove that (2.1) has an infinite number of roots and find their asymptotic formula. From (1.15), (1.17), (1.18), and (1.19) we have

$$
f^{\prime}(0, \lambda)-h f(0, \lambda)=b(\lambda)=f(1, \lambda) \varphi^{\prime}(1, \lambda)-f^{\prime}(1, \lambda) \varphi(1, \lambda) .
$$

Now, we calculate the asymptotic formula of $f(1, \lambda), f^{\prime}(1, \lambda), \varphi(1, \lambda)$ and $\varphi^{\prime}(1, \lambda)$. Integrating (1.15) by parts we have, for $x \geq 1, \operatorname{Im} \lambda>0$,

$$
\begin{aligned}
& f(x, \lambda)=e^{i \lambda x}-\frac{K(x, x)}{i \lambda} e^{i \lambda x}-\int_{x}^{\infty} \frac{e^{i \lambda x}}{i \lambda} \frac{\partial K(x, x)}{\partial t} d t \\
& f^{\prime}(x, \lambda)=i \lambda e^{i \lambda x}-K(x, x) e^{i \lambda x}+\int_{x}^{\infty} \frac{\partial K(x, x)}{\partial x} e^{i \lambda x} d t .
\end{aligned}
$$

Similarly from (1.18) we have

$$
\begin{aligned}
& \varphi(x, \lambda)=\cosh \lambda x+A(x, x) \frac{\sinh \lambda x}{\lambda}-\frac{1}{\lambda} \int_{0}^{x} \frac{\partial A(x, t)}{\partial t} \sinh \lambda t d t, \\
& \varphi^{\prime}(x, \lambda)=\lambda \sinh \lambda x+A(x, x) \cosh \lambda x+\int_{0}^{x} \frac{\partial A(x, t)}{\partial x} \cosh \lambda t d t .
\end{aligned}
$$

The following group of inequalities follows from (2.3)-(2.6):

$$
\begin{aligned}
& f(1, \lambda)=e^{i \lambda}+O\left(\frac{e^{-\operatorname{Im} \lambda}}{\lambda}\right), \\
& f^{\prime}(1, \lambda)=i \lambda e^{i \lambda}+O\left(e^{-\operatorname{Im} \lambda}\right), \\
& \varphi(1, \lambda)=\cosh \lambda+O\left(\frac{e^{|\operatorname{Re} \lambda|}}{\lambda}\right),
\end{aligned}
$$




$$
\varphi^{\prime}(1, \lambda)=\lambda \sinh \lambda+\mathrm{O}\left(e^{|\operatorname{Re} \lambda|}\right)
$$

Substituting (2.7)-(2.10) into (2.2), we obtain

$$
\begin{aligned}
& f^{\prime}(0, \lambda)-h f(0, \lambda)=-i \lambda e^{i \lambda}[\cosh \lambda+i \sinh \lambda]+\mathrm{O}\left(e^{-\operatorname{Im} \lambda+|\operatorname{Re} \lambda|}\right), \\
& \lambda \neq 0, \operatorname{Im} \lambda>0 ;
\end{aligned}
$$

comparing (1.10) and (2.11) we see that $f^{\prime}(0, \lambda)-h f(0, \lambda)$ and $f_{o}(0, \lambda)=e^{i \lambda}[\cosh \lambda+i \sinh \lambda]$ have the same number of zeros inside the quadratic contour $\Gamma_{n}$ where $\left\{\Gamma_{n}:|\operatorname{Re} \lambda| \leq \pi(n-\right.$ $\left.\left.\frac{1}{4}\right), 0<\operatorname{Im} \lambda \leq \pi\left(n-\frac{1}{4}\right)\right\}$, but since $f_{o}(0, \lambda)$ has exactly $n$ zeros, namely $\lambda_{k}^{o}=i \pi\left(k-\frac{1}{4}\right), k=$ $1,2, \ldots, n, f^{\prime}(0, \lambda)-h f(0, \lambda)$ has an infinite number of zeros, as $n \rightarrow \infty$, with limiting point at infinity. Denote by $\lambda_{n}$ the zeros of $f^{\prime}(0, \lambda)-h f(0, \lambda)=0$, so that, by the Rouche theorem, we have

$$
\lambda_{n}=i\left(n+\frac{1}{4}\right)+\varepsilon_{n}
$$

To make (2.12) more accurate, we must refine (2.11). With the aid of Lemma 1.1, $\lambda_{n}$ lies on the imaginary axis, so that it is sufficient to know the asymptotic of $f^{\prime}(0, \lambda)-h f(0, \lambda)$ for small $\lambda$. Let $\lambda=i \tau, \tau>0$, we find the asymptotic formula of $f^{\prime}(0, i \tau)-h f(0, i \tau)$ for $\tau \rightarrow \infty$. From (2.3), (2.4), (2.5), and (2.6), we have

$$
\begin{aligned}
& f(1, i \tau)=e^{-\tau}+K(1,1) \frac{e^{-\tau}}{\tau}+o\left(\frac{e^{-\tau}}{\tau}\right), \\
& 7 f^{\prime}(1, i \tau)=-\tau e^{-\tau}-K(1,1) e^{-\tau}+o\left(e^{-\tau}\right), \\
& \varphi(1, i \tau)=\cos \tau+A(1,1) \frac{\sin \tau}{\tau}+o\left(\frac{1}{\tau}\right), \\
& \varphi^{\prime}(1, i \tau)=-\tau \sin \tau+A(1,1) \cos \tau+o(1),
\end{aligned}
$$

substituting (2.13) into $f^{\prime}(0, i \tau)-h f(0, i \tau)=0$, and putting $\lambda_{n}=i \tau_{n}$ we have

$$
\cos \tau_{n}-\sin \tau_{n}+\frac{A(1,1)+K(1,1)}{\tau_{n}}\left(\cos \tau_{n}+\sin \tau_{n}\right)+o\left(\frac{e^{-\tau_{n}}}{\tau_{n}}\right)=0,
$$

and from this and by virtue of the inequality $\left|\cos \tau_{n}\right| \geq \delta>0 \forall n$, we have

$$
\begin{aligned}
1-\tan \tau_{n}+\frac{\alpha}{\tau_{n}}+\frac{\beta}{\tau_{n}} \tan +o\left(\frac{1}{\tau_{n}}\right) & =0 \\
\text { where } \alpha & =A(1,1)+K(1,1), \beta=A(1,1)-K(1,1) .
\end{aligned}
$$

From (2.12), it is easy to see that

$$
\begin{aligned}
& \tau_{n}=\left(n+\frac{1}{4}\right)+\varepsilon_{n}, \\
& \tan \tau_{n}=1+2 \varepsilon_{n}+\mathrm{O}\left(\frac{1}{n^{3}}\right), \\
& \frac{1}{\tau_{n}}=\frac{1}{n \pi}+\mathrm{O}\left(\frac{1}{n}\right) .
\end{aligned}
$$


The estimation of $\varepsilon_{n}$ follows from (2.15) and (2.16) in the form

$$
\varepsilon_{n}=-1+\frac{\beta-\alpha}{n \pi}+\mathrm{o}\left(\frac{1}{n}\right)
$$

Therefore

$$
\tau_{n}=\pi\left(n+\frac{1}{4}\right)-1+\frac{c_{o}}{n \pi}+o\left(\frac{1}{n}\right), \quad c_{o}=\frac{1}{\pi} \int_{0}^{1} q(t) d t .
$$

Finally

$$
\lambda_{n}=i\left[\pi\left(n+\frac{1}{4}\right)-1+\frac{c_{o}}{n \pi}+o\left(\frac{1}{n}\right)\right], \quad c_{o}=\frac{1}{\pi} \int_{0}^{1} q(t) d t .
$$

Definition (The normalizing numbers) The numbers

$$
a_{n} \stackrel{\text { def }}{=} \int_{0}^{\infty} \rho(x)\left|f\left(x, \lambda_{n}\right)\right|^{2} d x
$$

are called the normalizing numbers of problem (1.1)-(1.2) (notice that $f\left(x, \lambda_{n}\right)$ are the eigenfunctions of problem (1.1)-(1.2) corresponding to the eigenvalues $\lambda_{n}$ ). From (1.28) and the reality of $f\left(x, \lambda_{n}\right)$, we have

$$
a_{n} \stackrel{\text { def }}{=} \int_{0}^{\infty} \rho(x)\left|f\left(x, \lambda_{n}\right)\right|^{2} d x=-\frac{\left[\dot{f}^{\prime}\left(0, \lambda_{n}\right)-h \dot{f}\left(0, \lambda_{n}\right)\right] f\left(0, \lambda_{n}\right)}{2 \lambda_{n}} .
$$

To evaluate the asymptotic formula of $a_{n}$ we evaluate the asymptotic formula of the right hand side of (2.21). From (1.15), (1.17) we have

$$
\left[\dot{f}^{\prime}\left(0, \lambda_{n}\right)-h \dot{f}\left(0, \lambda_{n}\right)\right] f\left(0, \lambda_{n}\right)=[\dot{b}(\lambda)+(1+h) \dot{a}(\lambda)] a(\lambda),
$$

where dots and dashes denote the differentiation with respect to $\lambda$ and $\mathrm{x}$, respectively, $a(\lambda)$ and $b(\lambda)$ are given by (1.17)

$$
\begin{aligned}
& a(\lambda)=f(1, \lambda) \theta^{\prime}(1, \lambda)-f^{\prime}(1, \lambda) \theta(1, \lambda), \\
& b(\lambda)=f^{\prime}(1, \lambda) \varphi(1, \lambda)-f(1, \lambda) \varphi^{\prime}(1, \lambda),
\end{aligned}
$$

from which it follows that

$$
\begin{aligned}
& \dot{a}(\lambda)=\dot{f}(1, \lambda) \theta^{\prime}(1, \lambda)+f(1, \lambda) \dot{\theta}^{\prime}(1, \lambda)-\dot{f}^{\prime}(1, \lambda) \theta(1, \lambda)-f^{\prime}(1, \lambda) \dot{\theta}(1, \lambda), \\
& \dot{b}(\lambda)=\dot{f}^{\prime}(1, \lambda) \varphi(1, \lambda)+f^{\prime}(1, \lambda) \dot{\varphi}(1, \lambda)-\dot{f}(1, \lambda) \varphi^{\prime}(1, \lambda)-f(1, \lambda) \dot{\varphi}^{\prime}(1, \lambda) .
\end{aligned}
$$


From (1.18), using integration by parts and then putting $x=1, \lambda=i \tau$, we obtain

$$
\begin{aligned}
& \varphi(1, i \tau)=\cos \tau+A(1,1) \frac{\sin \tau}{\tau}+A_{t}(1,1) \frac{\cos \tau}{\tau^{2}}+o\left(\frac{1}{\tau^{2}}\right) \\
& \varphi^{\prime}(1, i \tau)=-\tau \sin \tau+A(1,1) \cos \tau+A_{t}(1,1) \frac{\sin \tau}{\tau}+o\left(\frac{1}{\tau}\right), \\
& \dot{\varphi}(1, i \tau)=-\sin \tau+A(1,1) \frac{\cos \tau}{\tau}-\left[A(1,1)+A_{t}(1,1)\right] \frac{\sin \tau}{\tau^{2}}+o\left(\frac{1}{\tau^{2}}\right), \\
& \dot{\varphi}^{\prime}(1, i \tau)=-\tau \cos \tau-[1+A(1,1)] \sin \tau-A_{t}(1,1) \frac{\cos \tau}{\tau}+A_{t}(1,1) \frac{\sin \tau}{\tau^{2}}+o\left(\frac{1}{\tau^{2}}\right) .
\end{aligned}
$$

From (1.19), carrying out a similar calculation with respect to $\theta$, we obtain

$$
\begin{aligned}
& l \theta(1, i \tau)=\frac{\sin \tau}{\tau}-B(1,1) \frac{\cos \tau}{\tau^{2}}+o\left(\frac{1}{\tau^{2}}\right), \\
& \theta^{\prime}(1, i \tau)=\cos \tau+B(1,1) \frac{\sin \tau}{\tau}+o\left(\frac{1}{\tau}\right), \\
& \dot{\theta}(1, i \tau)=\frac{\cos \tau}{\tau}-[1+B(1,1)] \frac{\sin \tau}{\tau^{2}}+2 B(1,1) \frac{\cos \tau}{\tau^{3}}+o\left(\frac{1}{\tau^{3}}\right), \\
& \dot{\theta}^{\prime}(1, i \tau)=-\sin \tau+B(1,1) \frac{\cos \tau}{\tau}-B(1,1) \frac{\sin \tau}{\tau^{2}}+o\left(\frac{1}{\tau^{2}}\right) .
\end{aligned}
$$

With the aid of (1.15), similar expressions can be calculated with respect to $f(1, i \tau)$ :

$$
\begin{aligned}
& f(1, i \tau)=e^{-\tau}+K(1,1) \frac{e^{-\tau}}{\tau}+o\left(\frac{e^{-\tau}}{\tau}\right), \\
& f^{\prime}(1, i \tau)=-\tau e^{-\tau}-K(1,1) e^{-\tau}+o\left(e^{-\tau}\right), \\
& \dot{f}(1, i \tau)=-e^{-\tau}-K(1,1) \frac{e^{-\tau}}{\tau}-K(1,1) \frac{e^{-\tau}}{\tau^{2}}+o\left(\frac{e^{-\tau}}{\tau^{2}}\right), \\
& \dot{f}^{\prime}(1, i \tau)=\tau e^{-\tau}-e^{-\tau}+K(1,1) e^{-\tau}+o\left(e^{-\tau}\right) .
\end{aligned}
$$

From (2.21) and (2.22), the normalizing numbers $a_{n}$ can be written in the form

$$
a_{n}=-\frac{[\dot{b}(\lambda)+(1+h) \dot{a}(\lambda)] a(\lambda)}{2 \lambda_{n}} .
$$

We substitute (2.23), (2.24), (2.25), and (2.26) into (2.27), $\lambda_{n}=i \tau_{n}$, and we find

$$
\begin{aligned}
a_{n}= & -e^{-2 \tau_{n}}\left\{\cos \tau_{n} \sin \tau_{n}+\cos ^{2} \tau_{n}+\frac{\alpha+\beta}{2 \tau_{n}} \cos \tau_{n} \sin \tau_{n}+\frac{\beta}{2 \tau_{n}} \sin ^{2} \tau_{n}\right. \\
& \left.+\frac{\alpha}{2 \tau_{n}} \cos ^{2} \tau_{n}+\frac{\alpha_{1}}{\tau_{n}} \cos \tau_{n} \sin \tau_{n}+\frac{\beta_{1}}{\tau_{n}} \cos ^{2} \tau_{n}+o\left(\frac{1}{\tau_{n}}\right)\right\}
\end{aligned}
$$


where $\alpha=1+2 K(1,1)+2 A(1,1), \beta=1+2 A(1,1), \alpha_{1}=B(1,1)+K(1,1)$, and $\beta_{1}=K(1,1)-$ $B(1,1)$. Further, from $(2.16)$ and $(2.17)$ we have

$$
\begin{aligned}
& \frac{1}{\tau_{n}}=\frac{1}{n \pi}\left[1+o\left(\frac{1}{\tau_{n}}\right)\right], \\
& \cos ^{2} \tau_{n}=\frac{3}{2}-\frac{\beta-\alpha}{n \pi}+o\left(\frac{1}{\tau_{n}}\right), \\
& \sin ^{2} \tau_{n}=\frac{1}{2}-\frac{\beta-\alpha}{n \pi}+o\left(\frac{1}{\tau_{n}}\right), \\
& \cos \tau_{n} \sin \tau_{n}=\frac{1}{2}\left[1+o\left(\frac{1}{\tau_{n}}\right)\right] .
\end{aligned}
$$

By substituting from (2.29) into (2.28) we obtain the required asymptotic formula for $a_{n}$ :

$$
a_{n}=-e^{-2 \tau_{n}}\left\{2+\frac{c_{1}}{n}+\mathrm{o}\left(\frac{1}{n}\right)\right\}
$$

where

$$
c_{1}=\frac{8 h+4}{4 \pi}+\frac{3}{8 \pi} \int_{0}^{1} q(t) d t+\frac{9}{8 \pi} \int_{1}^{\infty} q(t) d t .
$$

\section{Competing interests}

The authors declare that they have no competing interests.

\section{Authors' contributions}

The two authors typed read and approved the final manuscript also they contributed to each part of this work equally.

\section{Author details}

'Department of Mathematics, Faculty of Education, Alexandria University, Alexandria, Egypt. ${ }^{2}$ Department of Mathematics, Faculty of Industrial Education, Helwan University, Cairo, Egypt.

\section{Acknowledgements}

We are indebted to an anonymous referee for a detailed reading of the manuscript and useful comments and suggestions, which helped us improve this work. This work is supported by the Research Support Unit of Alexandria University.

Received: 30 January 2014 Accepted: 1 April 2014 Published: 06 May 2014

\section{References}

1. Everitt, WN: Charles Sturm and the development of Sturm-Liouville theory in the years 1900 to 1950. In: Sturm-Liouville Theory, pp. 271-331. Birkhäuser, Basel (2005)

2. Levitan, BM: Inverse Sturm-Liouville Problems. Nauka, Moscow (1984). English transl., VNU Sci. Press, Utrecht (1987)

3. Alimov, SA: The work of A.N. Tikhonov on inverse problems for the Sturm-Liouville equation. Usp. Mat. Nauk 31(6(192)), 84-88. (1976). English transl., Russ. Math. Surv. 31(6), 87-92 (1976)

4. Marchenko, VA: Sturm-Liouville Operators and Their Applications. Naukova Dumka, Kiev (1977). English transl., Birkhäuser (1986)

5. Tikhonov, AN: On the uniqueness of the solution of electric investigation problem. Doklady of the USSR 69(6), 797-800 (1949) (in Russian)

6. Gasymov, MG: Direct and inverse problems of spectral analysis for a class of equations with discontinuous coefficients. In: Proceedings of the International Conference on Non-Classical Methods in Geophysics, Novosibirsk, pp. 37-44. (1977) (in Russian)

7. Aliev, AR, Mirzoev, SS: On boundary value problem solvability theory for a class of high-order operator-differential equations. Funkc. Anal. Prilozh. 44(3), 63-65 (2010). English transl., Funct. Anal. Appl. 44(3), 209-211 (2010)

8. Mirzoev, SS, Aliev, AR, Rustamova, RA: Solvability conditions for boundary-value problems for elliptic operator-differential equations with discontinuous coefficient. Mat. Zametki 92(5), 789-793 (2012). English transl., Math. Notes 92(5), 722-726 (2012)

9. Mirzoev, SS, Aliev, AR, Rustamova, RA: On the boundary value problem with the operator in boundary conditions for the operator-differential equation of second order with discontinuous coefficients. J. Mat. Fiz. Anal. Geom. 9(2), 207-226 (2013) 
10. Aliev, AR: On the generalized solution of the boundary-value problem for the operator-differential equations of the second order with variable coefficients. J. Mat. Fiz. Anal. Geom. 2(1), 87-93 (2006)

11. Aliev, AR, Gasymova, SG, Gasymova, DG, Ahmadzadeh, ND: Approximate construction of the Jost function by the collocation method for Sturm-Liouville boundary value problem. Azerb. J. Math. 3(2), 45-61 (2013)

12. Freiling, G, Yurko, VA: Inverse Sturm-Liouville Problems and Their Applications. Nova Science Publishers, New York (2001)

13. Efendiev, RF, Orudzhev, HD: Inverse wave spectral problem with discontinuous wave speed. J. Mat. Fiz. Anal. Geom 6(3), 255-265 (2010)

14. Efendiev, RF: Spectral analysis for one class of second-order indefinite non-self-adjoint differential operator pencil. Appl. Anal. 90(12), 1837-1849 (2011)

15. El-Raheem, ZFA, Nasser, AH: On the spectral property of a Dirichlet problem with explosive factor. Appl. Math. Comput. 138(2-3), 355-374 (2003)

16. Gasymov, MG, Kakhramanov, AS, Petrosyan, SK: On the spectral theory of linear differential operators with discontinuous coefficients. Akad. Nauk Azerbaĭdzhan. SSR Dokl. 43(3), 13-16 (1987) (in Russian)

10.1186/1687-2770-2014-97

Cite this article as: El-Raheem and Nasser: On the spectral investigation of the scattering problem for some version of one-dimensional Schrödinger equation with turning point. Boundary Value Problems 2014, 2014:97

\section{Submit your manuscript to a SpringerOpen ${ }^{\circ}$ journal and benefit from:}

- Convenient online submission

Rigorous peer review

- Immediate publication on acceptance

- Open access: articles freely available online

- High visibility within the field

- Retaining the copyright to your article 